\title{
UNIVERSAL FUNCTIONS AND GENERALIZED CLASSES OF FUNCTIONS
}

\author{
J. CICHOŃ AND M. MORAYNE
}

(Communicated by J. Jerry Uhl, Jr.)

\begin{abstract}
For a class $A$ of subsets of a set $Z$ which is closed under countable unions we consider the families of functions

$$
\underline{M} A=\left\{f: Z \rightarrow[0,1]:(\forall c)\left(f^{-1}((c, 1]) \in A\right)\right\}
$$

and

$$
\bar{M} A=\left\{f: Z \rightarrow[0,1]:(\forall c)\left(f^{-1}([0, c)) \in A\right)\right\}
$$

(for instance, if $Z$ is a topological space and $A$ is the family of all open subsets of $Z$, then $\underline{M} A$ and $\bar{M} A$ are the families of lower and upper semicontinuous functions from $Z$ to $[0,1]$, respectively).

Using universal functions we show that under certain natural assumptions about $A$ there exists a function $f \in \underline{M} A$ such that there is no partition $\left\{X_{n}: n \in \mathbf{N}\right\}$ of $Z$ and a family of functions $\left\{h_{n}: n \in \mathbf{N}\right\} \subseteq \bar{M} A$ such that $f=\bigcup_{n}\left(h_{n} \mid X_{n}\right)$.

This is a generalization of some results of this type proved by Novikov and Adian, Keldys, and Laczkovich for the Baire hierarchy of functions. The universal functions technique we use is different from the methods of these authors.
\end{abstract}

1. Introduction. We use standard set theoretical notations. For example, if $A$ and $B$ are sets, then $P(A)$ is the set of all subsets of $A, A \times B$ is the Cartesian product of $A$ and $B$ and ${ }^{A} B$ is the family of all functions from $A$ to $B$. A family $S \subseteq P(A)$ is a partition of $A$ if $\bigcup S=A$ and for any different $X, Y \in S$ we have $X \cap Y=\varnothing$. By $I$ we always denote the closed interval $[0,1] . \mathbf{N}$ is the set of natural numbers and $\mathbf{Q}$ is the set of rational numbers.

Suppose that $A \subseteq P(Z)$ is a family of sets. We say that $A$ is a $\sigma$-class if $\{\varnothing, Z\} \subseteq A, A$ is closed under finite intersections, and for any countable $A^{\prime} \subseteq A$ we have $\bigcup A^{\prime} \in A$. By $\underline{M} A$ we denote the family of all functions $f \in{ }^{Z} I$ such that for any $c \in I$ we have $f^{-1}((c, 1]) \in A$. Similarly, $\bar{M} A$ is the family of all functions $f \in{ }^{Z} I$ such that for any $c \in I$ we have $f^{-1}([0, c)) \in A$. We also put $M A=\underline{M} A \cap \bar{M} A$. Note that $f \in \underline{M} A$ if and only if $1-f \in \bar{M} A$. If $X \subseteq Z$, then $A \mid X=\{A \cap X: A \in A\}$. By $(G \underline{M}) A$ we denote the family of all functions $f \in{ }^{Z} I$ such that there exists a partition $\left\{X_{n}\right\}_{n \in \mathbf{N}}$ of $Z$ such that for any $n \in \mathbf{N}$ we have $f \mid X_{n} \in \underline{M}\left(A \mid X_{n}\right)$. Similarly we define $(G \bar{M}) A$ and $(G M) A$ (this means that we replace $\underline{M} A$ by $\bar{M} A$, or $M A$, in the above definition).

Received by the editors November 30, 1984 and, in revised form, September 3, 1985.

1980 Mathematics Subject Classification (1985 Revision). Primary 26A21; Secondary 04A15, 26A15.

Key words and phrases. Generalized classes of functions, universal functions, Baire classes. 
Proposition 1.1. If $A$ is a $\sigma$-class of subsets of $Z, X \subseteq Z$ and $f \in \underline{M}(\mathcal{A} \mid X)$, then there is $f^{*} \in \underline{M} A$ such that $f \subseteq f^{*}$.

ProOF. Suppose that $f \in \underline{M}(A \mid X)$. For any rational number $q \in \mathbf{Q} \cap I$ we have $f^{-1}((q, 1]) \in A \mid X$. Hence there exists a set $A_{q} \in A$ such that $f^{-1}((q, 1])=A_{q} \cap X$. For $x \in A_{q}$ we put $f_{q}(x)=q$ and $f_{q}(x)=0$ otherwise. Then for any $q \in \mathbf{Q} \cap I$ we have $f_{q} \in \underline{M} A$. Let $f^{*}=\sup \left\{f_{q}: q \in I \cap \mathbf{Q}\right\}$. Then, as $\mathcal{A}$ is closed under countable unions, $f^{*} \in \underline{M}$ A. It is clear that $f \subseteq f^{*}$.

The functions $f \in \bar{M} A$ have a similar property. Namely, if $A \subseteq P(Z)$ is a $\sigma$-class, $X \subseteq Z$ and $f \in \bar{M}(A \mid X)$, then for some $f^{*} \in \bar{M} A$ we have $f \subseteq f^{*}$. In order to see this, suppose that $f \in \bar{M}(A \mid X)$. Then $1-f \in \underline{M}(A \mid X)$, Hence we can find an extension of $1-f$ to a function $f_{1} \in \underline{M} A$. Then $1-f_{1} \in \bar{M} A$ is the required function.

For any family $\not \subset \subseteq{ }^{Z} I$ let $G \not{H}$ be the family of all functions $f \in{ }^{Z} I$ such that there is a partition $\left\{X_{n}\right\}_{n \in \mathbf{N}}$ of $Z$ and a family of functions $\left\{f_{n}\right\}_{n \in \mathbf{N}} \subseteq \not{H}$ such that for any $n \in \mathbf{N}$ we have $f\left|X_{n}=f_{n}\right| X_{n}$.

COROLlaRY 1.2. If $A$ is a $\sigma$-class, then $(G \underline{M}) A=G(\underline{M} A)$.

PROOF. The inclusion $G(\underline{M} A) \subseteq(G \underline{M}) A$ is true for any class $A$. The second inclusion follows from Proposition 1.1.

Notice that we also have $(G \bar{M}) \mathcal{A}=G(\bar{M} A)$ for any $\sigma$-class $A$ and that equality $(G M) A=G(M A)$ is not generally true.

Proposition 1.3. For any $\sigma$-class $A$ of subsets of $Z$ the family $\underline{M} A$ is closed under uniform convergence.

PROOF. If $\left\{f_{n}\right\}_{n \in \mathbf{N}} \subseteq \underline{M} A$ is uniformly convergent to a function $f$, then for any $c \in I$ we have

$$
f^{-1}((c, 1])=\bigcup_{n=1}^{\infty} f_{n}^{-1}\left(\left(c+\sup \left\{\left|f(z)-f_{n}(z)\right|: z \in Z\right\}, 1\right]\right) .
$$

By $C$ we denote the Cantor set ${ }^{\mathbf{N}}\{0,1\}$ with the product topology. Recall that a Polish space is a separable complete metric space. We consider the hierarchy of Borel subsets of Polish spaces. $\sum_{\alpha}^{0}(X)$ is the $\alpha$ th additive class of subsets of $X$ where $\alpha<\omega_{1} \cdot \sum_{n}^{1}(X), n \in \mathbf{N}$, denote the projective hierarchy of subsets of $X$.

Let us agree to consider throughout this paper a certain class $\mathcal{F}$ of Polish spaces such that a finite product of elements of $\mathcal{F}$ is again in $\mathcal{F}$ and if $Z \in \mathcal{F}$ then for each Polish space $X \subseteq Z$ ( $X$ has the metric of $Z$ ) $X$ is also in $₹$. Let us also assume that $C, I \in \mathcal{F}$. In fact the idea is to include in $\mathcal{F}$ any Polish space one wants to consider (this idea was used in [6]).

Let us assume that we have defined a certain family of subsets $A$ on each $Z \in \mathcal{F}$ simultaneously. $A$ defined on subsets of a specific $Z \in \mathcal{F}$ will be denoted by $A(Z)$. We say that $A$ is closed under continuous substitution if for each $X, Y \in \mathcal{F}$, for every continuous function $f \in{ }^{X} Y$ and for each $A \in A(Y)$ we have $f^{-1}(A) \in A(X)$. $A$ will be called a hereditary $\sigma$-class if $A$ is closed under continuous substitution and if for each $Z \in \mathcal{F}$ :

(i) $A(Z)$ is a $\sigma$-class,

(ii) $A(Z) \mid X=A(X)$ for each Polish space $X \subseteq Z$. 
The classes $\sum_{\alpha}^{0}, \alpha<\omega_{1}$, and $\sum_{n}^{1}, n \in \mathbf{N}$, are examples of hereditary $\sigma$-classes.

For $A \subseteq X \times Y$ and $x \in X$ let $A_{x}=\{y \in Y:\langle x, y\rangle \in A\}$. Let $A \subset P(Y)$. A set $A \subseteq X \times Y$ is a universal set for $A$ if $A=\left\{A_{x}: x \in X\right\}$. Recall that if $X$ and $Y$ are Polish spaces and $X$ is uncountable, then for any $\alpha<\omega_{1}$ there is a universal set for $\sum_{\alpha}^{0}(Y)$ in the class $\sum_{\alpha}^{0}(X \times Y)$ and the same is true for the classes $\sum_{n}^{1}, n \in \mathbf{N}$ (see [6]).

2. Universal functions. For a function $F \in{ }^{X \times Y} I$ and $\langle x, y\rangle \in X \times Y$ we put $F_{x}(y)=F(x, y)$. Suppose that $\sharp \subseteq{ }^{Y} I$. A function $F \in{ }^{X \times Y} I$ is called a universal function for $\not$ if $\mathcal{H}=\left\{F_{x}: x \in X\right\}$.

THEOREM 2.1. Let $A$ be a hereditary $\sigma$-class. If $Z \in \mathcal{F}$ is uncountable and there exists a universal set for $A(Z)$ in $A(C \times Z)$, then there exists a universal function for $\underline{M} A(Z)$ in $\underline{M} A\left(Z^{2}\right)$.

ProOF. First we prove that there exists a universal function for $\underline{M} A(Z)$ in $\underline{M} A(C \times Z)$.

For any $X \subseteq Z$ let

$$
\chi(X, z)= \begin{cases}0 & \text { if } z \in Z \backslash X \\ 1 & \text { if } z \in X .\end{cases}
$$

Let $\phi=\left(\phi_{1}, \phi_{2}, \ldots\right)$ be a homeomorphism of $C$ and ${ }^{\mathbf{N}} C$. Let $A \in A(C \times Z)$ be a universal set for $A(Z)$. We fix an enumeration $\left\{r_{n}\right\}_{n \in \mathbf{N}}$ of $\mathbf{Q} \cap I$.

For any $n \in \mathbf{N}$ and $\langle c, z\rangle \in C \times Z$ we put $F_{n}(c, z)=\chi\left(A_{\phi_{n}(c)}, z\right)$ and $\bar{\phi}_{n}(c, z)=$ $\left\langle\phi_{n}(c), z\right\rangle$. Obviously, $\bar{\phi}_{n}$ is a continuous function and for any $n \in \mathbf{N}$ the function $F_{n}$ is the characteristic function of the set $\bar{\phi}_{n}^{-1}(A)$. Hence $F_{n} \in \underline{M} A(C \times Z)$, for $A$ is closed under continuous substitution.

Let $F(c, z)=\sup \left\{r_{n} \cdot F_{n}(c, z): n \in \mathbf{N}\right\}$. Then for any $c \in C$ we have $F_{c} \in \underline{M} A(Z)$ and $F \in \underline{M} A(C \times Z)$. Suppose that $f \in \underline{M} A(Z)$. For any $n \in \mathrm{N}$ let $c_{n} \in C$ be such that $A_{c_{n}}=\left\{z \in Z: f(z)>r_{n}\right\}$. Let $c \in \mathcal{C}$ be such that $\phi(c)=\left\langle c_{1}, c_{2}, \ldots\right\rangle$. Then we have $F_{c}=f$. Hence $F$ is a universal function for $\underline{M} A(Z)$.

Because we can embed $C$ into any uncountable Polish space and because $A$ is closed under continuous substitution, we can consider $C$ here as a compact subset of $Z$.

We have $A(C \times Z)=A\left(Z^{2}\right) \mid C \times Z$ whence, by Proposition 1.1, there exists an extension $F^{*} \in \underline{M} A\left(Z^{2}\right)$ of $F$. For every $z \in Z$ the function $F_{z}^{*}$ belongs to $\underline{M} A(Z)$, because $A$ is closed under continuous substitution. Thus $F^{*}$ is universal for $\underline{M} A(Z)$.

3. Generalized classes of functions. N. N. Lusin asked the question whether $M\left(\bigcup_{\xi<\omega_{1}} \sum_{\xi}^{0}\right) \subseteq(G M) \sum_{1}^{0}$. This question was answered negatively by $\mathrm{P}$. S. Novikov (see [2]). Later S. I. Adian and P. S. Novikov showed in [1] ([7]) that

$$
\bar{M} \sum_{1}^{0}(I) \backslash(G M) \sum_{1}^{0}(I) \neq \varnothing .
$$

It was shown by L. Keldys in [2] that

$$
M \sum_{\alpha+1}^{0}(\mathcal{N}) \backslash G\left(\bigcup_{\xi<\alpha} M \sum_{\xi+1}^{0}(\mathcal{N})\right) \neq \varnothing
$$

for any $\alpha<\omega_{1}$, where $\mathcal{N}={ }^{\mathbf{N}} \mathbf{N}$ is the space of irrational numbers. Quite recently M. Laczkovich proved that for each $\alpha<\omega_{1}$ and for any uncountable Polish space 
$Z$ there exists a function $f \in M \sum_{\alpha}^{0}(Z)$ such that for no partition $Z=\bigcup_{n=1}^{\infty} X_{n}$ the functions $f \mid X_{n}$ are of class $M \sum_{\alpha_{n}}^{0}\left(X_{n}\right)$ where $\alpha_{n}<\alpha$, for each $n \in \mathbf{N}$. His proof gives for any $\alpha<\omega_{1}[\mathbf{4}]$ :

$$
\underline{M} \sum_{\alpha}^{0}(Z) \backslash(G M) \sum_{\alpha}^{0}(Z) \neq \varnothing .
$$

We prove (Corollary 3.3) that if a class $A$ satisfies the assumptions of Theorem 2.1 and $Z$ is an uncountable Polish space, then $\underline{M} A(Z) \backslash(G \bar{M}) A(Z) \neq \varnothing$. Note that this is equivalent to $\underline{M} A(Z) \backslash(G M) A(Z) \neq \varnothing$ by the definition of $M A$. This gives in particular $\underline{M} \sum_{\alpha}^{0}(Z) \backslash(G M) \sum_{\alpha}^{0}(Z) \neq \varnothing$.

THEOREM 3.1. If $A$ is a $\sigma$-class of subsets of $Z$ and $f \in(G \bar{M}) \mathcal{A}$, then there exists a function $g \in \underline{M} A$ such that for any $z \in Z$ we have $f(z) \neq g(z)$.

PROOF. For any $k \in \mathbf{N}$ and for any sequence $n_{1}, \ldots, n_{k} \in \mathbf{N}$ we choose numbers $a\left(n_{1}, \ldots, n_{k}\right) \in(0,1)$ and $\varepsilon\left(n_{1}, \ldots, n_{k}\right)>0$ in such a way that the following conditions hold:

(i) the sequence $\{a(n)\}_{n \in \mathbf{N}}$ is strictly increasing and tends to 1 ;

(ii) for any $n_{1}, \ldots, n_{k}$ the sequence $\left\{a\left(n_{1}, \ldots, n_{k}, n\right)\right\}_{n \in \mathbf{N}}$ is strictly increasing and tends to $a\left(n_{1}, \ldots, n_{k}\right)$;

(iii) for any $k, m \in \mathbf{N}, k<m$, and $n_{1}, \ldots, n_{m} \in \mathbf{N}$ the following inequality holds:

$$
a\left(n_{1}, \ldots, n_{k}\right)+\varepsilon\left(n_{1}, \ldots, n_{k}\right)<a\left(n_{1}, \ldots, n_{k-1}, n_{k}+1, n_{k+1}, \ldots, n_{m}\right) ;
$$

(iv) for any $n_{1}, \ldots, n_{k} \in \mathbf{N}$ we have

$$
a\left(n_{1}, \ldots, n_{k}\right)-a\left(n_{1}, \ldots, n_{k}, 1\right)<1 / k .
$$

It is easy to see that such a choice is possible.

We have assumed that $f \in(G \bar{M}) A(Z)=G(\bar{M} A(Z)$ ) (Corollary 1.2) whence there is a partition $\left\{A_{n}: n \in \mathbf{N}\right\}$ of $Z$ and a family $\left\{f_{n}: n \in \mathbf{N}\right\} \subseteq \bar{M} A$ such that $f_{n}\left|A_{n}=f\right| A_{n}$ for each $n \in \mathbf{N}$.

Let $S(1)=\left\{z \in Z: f_{1}(z) \geq a(1)+\varepsilon(1)\right\}$ and for $n>1$

$$
S(n)=\left\{z \in Z: f_{n}(z) \geq a(n)+\varepsilon(n)\right\} \backslash \bigcup\left\{S_{i}: i<n\right\} .
$$

Suppose now that the set $S\left(n_{1}, \ldots, n_{k}\right)$ has been defined. Then for $n \leq n_{k}$ we put $S\left(n_{1}, \ldots, n_{k}, n\right)=\varnothing$ and for $n>n_{k}$ we define

$$
\begin{aligned}
S\left(n_{1}, \ldots, n_{k}, n\right)= & S\left(n_{1}, \ldots, n_{k}\right) \\
& \cap\left\{z \in Z: f_{n}(z) \geq a\left(n_{1}, \ldots, n_{k}, n\right)+\varepsilon\left(n_{1}, \ldots, n_{k}, n\right)\right\} \\
& \backslash \bigcup\left\{S\left(n_{1}, \ldots, n_{k}, i\right): i<n\right\} .
\end{aligned}
$$

Let $g_{1}(z)=a(n)$ if $z \in S(n)$ for some $n \in \mathbf{N}$ and $g_{1}(z)=1$ otherwise.

Suppose that $g_{k}$ is defined. Then we put $g_{k+1}(z)=a\left(n_{1}, \ldots, n_{k+1}\right)$ if $z \in$ $S\left(n_{1}, \ldots, n_{k+1}\right)$ for some $n_{1}, \ldots, n_{k+1} \in N$ and $g_{k+1}(z)=g_{k}(z)$ otherwise. 
Let $c \in[0,1)$ and $k \in \mathbf{N}$. We define inductively a sequence $n_{1}, n_{2}, \ldots$ as follows: $n_{1}=\min \{n: c<a(n)\}, n_{k}=\min \left\{n: c<a\left(n_{1}, \ldots, n_{k-1}, n\right)\right\}$. Then

$$
\begin{aligned}
g_{k}^{-1}((c, 1])= & \left(\bigcup_{i=1}^{n_{1}-1} f_{i}^{-1}([a(i)+\varepsilon(i), 1])\right. \\
& \cup \bigcup_{j=1}^{k-1}\left(\bigcap_{i=1}^{j} f_{n_{i}}^{-1}\left(\left[a\left(n_{1}, \ldots, n_{i}\right)+\varepsilon\left(n_{1}, \ldots, n_{i}\right), 1\right]\right)\right. \\
& \left.\left.\cap \bigcup_{r=n_{j}+1}^{n_{j+1}-1} f_{r}^{-1}\left(\left[a\left(n_{1}, \ldots, n_{j}, r\right)+\varepsilon\left(n_{1}, \ldots, n_{j}, r\right)\right]\right)\right)\right)^{c} .
\end{aligned}
$$

Hence $g_{k} \in \underline{M} A$. Moreover condition (iv) ensures that the sequence $\left\{g_{k}: k \in \mathbf{N}\right\}$ is uniformly convergent. Let $g=\lim _{k} g_{k}$. Then by Proposition 1.3 we have $g \in \underline{M} A$. We shall show that $f(z) \neq g(z)$ for any $z \in Z$.

Assume that for some $z \in Z$ we have $f(z)=g(z)$. Let us fix $k \in \mathbf{N}$ such that $z \in A_{k}$.

Note that we must have $z \in \bigcup\{S(n): n \in \mathbf{N}\}$, because otherwise $g(z)=1$ and $f(z)<a(k)<1$. Moreover, note that $z \in \bigcup\{S(n): n<k\}$, because otherwise either $z \in S(k)$, whence $f(z)=f_{k}(z) \geq a(k)+\varepsilon(k)$ and $g(z) \leq a(k)$, or $z \in S(n)$ for some $n>k$, whence $f(z)=f_{k}(z)<a(k)+\varepsilon(k)$ and $g(z) \geq a(k)+\varepsilon(k)$.

Hence there exists $n_{1}<k$ such that $z \in S\left(n_{1}\right)$.

Using the same argument we can find $n_{2}<k$ such that $z \in S\left(n_{1}, n_{2}\right)$. Iterating this procedure we define a sequence $\left\{n_{j}\right\}_{j \in \mathbf{N}}$ such that for any $m \in \mathbf{N}$ we have $n_{m}<$ $k$ and $z \in S\left(n_{1}, \ldots, n_{m}\right)$. But this gives $n_{1}<n_{2}<\cdots$, because $S\left(n_{1}, \ldots, n_{m}\right) \neq \varnothing$ implies $n_{1}<n_{2}<\cdots<n_{m}$. This is a contradiction. Hence the function $g$ has the required properties.

We prove now the main theorem of this paper.

THEOREM 3.2. Let $A$ be a $\sigma$-class of subsets of $Z$ and let $F \in{ }^{Z \times Z} I$ be a universal function for $\underline{M} A$. Let $\phi(z)=F(z, z)$ for $z \in Z$. Then $\phi \notin(G \bar{M}) A$.

ProOF. Suppose that $\phi \in(G \bar{M}) A$. By Theorem 3.1 there exists $f \in \underline{M} A$ such that for any $z \in Z$ we have $\phi(z) \neq f(z)$. Let $z_{0} \in Z$ be such that $f=F_{z_{0}}$. Then we have $f\left(z_{0}\right)=F_{z_{0}}\left(z_{0}\right)=F\left(z_{0}, z_{0}\right)=\phi\left(z_{0}\right) \neq f\left(z_{0}\right)$. This gives a contradiction.

By Theorem 2.1 we obtain the following corollary.

COROLLARY 3.3. If $A$ is a hereditary $\sigma$-class, $Z \in \mathcal{F}$ is uncountable and there exists a universal set for $A(Z)$ in $A(C \times Z)$, then $\underline{M} A(Z) \backslash(G \bar{M}) A(Z) \neq \varnothing$.

In particular the following holds:

Corollary 3.4. If $Z$ is any uncountable Polish space, then

$$
\underline{M} \sum_{\alpha}^{0}(Z) \backslash(G \bar{M}) \sum_{\alpha}^{0}(Z) \neq \varnothing
$$

for every $\alpha<\omega_{1}$ and $\underline{M} \sum_{n}^{1}(Z) \backslash(G \bar{M}) \sum_{n}^{1}(Z) \neq \varnothing$ for every $n \in \mathbf{N}$.

4. Additional remarks. A set $X \subseteq I$ is a Lusin set if for any first Baire category set $A \subseteq I$ we have $|A \cap X|<2^{\omega}$ and $|X|=2^{\omega}$. 
Sierpiński in [8] showed that if there exists a Lusin set, then there exists a function $f \in \bar{M} \sum_{1}^{0}(I)$ and a set $E \subseteq I$ such that $|E|=2^{\omega}$ and for any $F \subseteq E$ of cardinality $2^{\omega}$ the function $f \mid F$ is not continuous.

Let us recall that the Continuum Hypothesis as well as Martin's Axiom imply that there exists a Lusin set.

COROLLARY 4.1. Suppose that Martin's Axiom holds. Then there exists a function $f \in \bar{M} \sum_{1}^{0}(I)$ such that for any family $\&$ consisting of continuous functions on their domains if $\bigcup H=f$, then $|H|=2^{\omega}$.

PROOF. Let $f \in \bar{M} \sum_{1}^{0}$ and $E \subseteq I$ be such that $|E|=2^{\omega}$ and for every $F \subseteq E$, if $|F|=2^{\omega}$, then $f \mid F$ is not continuous. Suppose that $\bigcup \mathcal{H}=f$ and $|\mathcal{H}|<2^{\omega}$. Then there exists $h_{0} \in \not /$ such that $\mid E \cap$ domain $\left(h_{0}\right) \mid=2^{\omega}$. Therefore $h_{0}$ is not a continuous function.

We shall show later that the assumption about Lusin sets in Sierpiński's result is necessary. But before this we show that under the Continuum Hypothesis we can generalize Sierpiński's result to wider classes of functions.

THEOREM 4.2. Assume the Continuum Hypothesis. Let $A \subseteq P(Z)$ be a $\sigma$-field, $|A| \leq 2^{\omega}, \not \forall \cup\{f\} \subseteq M A$ and $f \notin G \not$. Then there exists $E \subseteq Z,|E|=2^{\omega}$, such that for every $h \in \mathcal{H}$ we have $|\{z \in E: h(z)=f(z)\}|<2^{\omega}$.

ProOF. Let $I$ be a family of subsets of $Z$ defined as follows:

$X \in I \quad$ if and only if $\left(\exists\left\{A_{n}: n \in \mathbf{N}\right\} \subseteq P(Z)\right)\left(\exists\left\{h_{n}: n \in \mathbf{N}\right\} \subseteq \mathcal{H}\right)$

$\left(X \subseteq \bigcup_{n} A_{n}\right.$ and $\left.(\forall n \in \mathbf{N})\left(f\left|A_{n}=h_{n}\right| A_{n}\right)\right)$.

Note that $I$ is a proper $\sigma$-ideal of subsets of $Z$. Moreover, for any $X \in I$ we can find $A \in I \cap A$ such that $X \subseteq A$.

Let $I \cap \mathcal{A}=\left\{A_{\alpha}: \alpha<\omega_{1}\right\}$. For any $\alpha<\omega_{1}$ we choose a point

$$
e_{\alpha} \in Z \backslash \bigcup_{\xi<\alpha}\left(A_{\xi} \cup\left\{e_{\xi}\right\}\right)
$$

and we put $E=\left\{e_{\alpha}: \alpha<\omega_{1}\right\}$.

Suppose now that $h \in \mathcal{H}$. Then $\{z \in Z: h(z)=f(z)\} \in I \cap \mathcal{A}$. Hence there is $\alpha<\omega_{1}$ such that $\{z \in Z: h(z)=f(z)\}=A_{\alpha}$. Thus $\{z \in E: h(z)=f(z)\} \subseteq$ $\left\{e_{\xi}: \xi \leq \alpha\right\}$.

Let $\mathfrak{d}$ denote the minimal cardinality of a family $\sharp \subseteq{ }^{\mathbf{N}} \mathbf{N}$ such that for any $f \in{ }^{\mathbf{N}} \mathbf{N}$ there is $g \in \mathcal{H}$ satisfying the inequality $f(n) \leq \bar{g}(n)$ for each $n \in \mathbf{N}$. It is well known that the theory $\mathrm{ZFC}+\left(2^{\omega}=\omega_{2}\right)+\left(\mathfrak{d}=\omega_{1}\right)$ is consistent (see [5]).

THEOREM 4.3. For any function $f \in M \sum_{2}^{1}(I)$ there is a family $\not$ of continuous functions such that $|\mathcal{H}| \leq \mathfrak{O}$ and $\bigcup \mathcal{H}=f$.

PROOF. Note that if $f \in M \sum_{2}^{1}(I)$ then $f \in \sum_{2}^{1}\left(I^{2}\right)$ (treated as subset of $\left.I \times I\right)$. Hence there exists a sequence $\left\{B_{\alpha}\right\}_{\alpha<\omega_{1}}$ of Borel sets such that $f=\bigcup_{\alpha<\omega_{1}} B_{\alpha}$ (see [3, p. 391]). Since any Borel set is an analytic set, we can find for any $\alpha<\omega_{1}$ a continuous function $f_{\alpha}$ from $\mathcal{N}={ }^{\mathbf{N}} \mathbf{N}$ onto $B_{\alpha}$.

Let $\left\{h_{\beta}: \beta<\mathfrak{d}\right\} \subseteq{ }^{\mathbf{N}} \mathbf{N}$ be such that for any $f \in{ }^{\mathbf{N}} \mathbf{N}$ there exists $\beta<\mathfrak{d}$ such that $f(n) \leq h_{\beta}(n)$ for each $n \in \mathbf{N}$. For any $\beta<\mathfrak{d}$ let $K_{\beta}=\left\{f \in{ }^{\mathbf{N}} \mathbf{N}: f(n) \leq h_{\beta}(n)\right.$ for each $n \in \mathbf{N}\}$. Note that for any $\beta<\mathfrak{d}$ the set $K_{\beta}$ is a compact subset of ${ }^{\mathbf{N}} \mathbf{N}$. 
For $\alpha<\omega_{1}$ and $\beta<\mathfrak{d}$ we put $B_{\alpha, \beta}=f_{\alpha}\left(K_{\beta}\right)$ and $\not H=\left\{B_{\alpha, \beta}: \alpha<\omega_{1}\right.$ and $\beta<\mathfrak{d}\}$. Then $|\mathcal{H}| \leq \mathfrak{d}$ and $\bigcup \mathcal{H}=f$. Moreover, if $\alpha<\omega_{1}$ and $\beta<\mathfrak{d}$, then $B_{\alpha, \beta}$ is a compact set. Hence $\not$ consists of continuous functions.

COROLlARY 4.4. Suppose that $2^{\omega}=\omega_{2}$ and $\mathfrak{d}=\omega_{1}$. Then for every function $f \in M \sum_{2}^{1}(I)$ and a set $E \subseteq I$ of cardinality $2^{\omega}$ there exists $F \subseteq E$ such that $|F|=2^{\omega}$ and $f \mid F$ is continuous.

ProOF. Let $f \in M \sum_{2}^{1}(I), E \subseteq I$, and $|E|=2^{\omega}$. Theorem 4.3 implies that there is a family $\not H$ consisting of continuous functions such that $\bigcup \forall=f$ and $|\not H|=\omega_{1}$. Then there exists a function $h \in \mathcal{H}$ such that $|\operatorname{domain}(h) \cap E|=\omega_{2}$.

ACKNOWLEDGMENT. We wish to thank Professor Czesław Ryll-Nardzewski for drawing our attention to the subject and for his valuable remarks concerning the paper.

\section{REFERENCES}

1. S. I. Adian and P. S. Novikov, On one semicontinuous function, Uchen. Zap. MGPI W. I. Lenina 138 (3) (1958), 3-10. (Russian)

2. L. Keldys, Sur les fonctions premières mesurables B, Dokl. Akad. Nauk SSSR 4 (1934), 192197. (Russian and French)

3. K. Kuratowski, Topologie I, PWN, Warszawa, 1958.

4. M. Laczkovich, unpublished preprint.

5. A. W. Miller, Some properties of measure and category, Trans. Amer. Math. Soc. 266 (1981), 93-114.

6. Y. N. Moschovakis, Descriptive set theory, North-Holland, Amsterdam, 1980.

7. P. S. Novikov, Collected papers. Set and function theory. Mathematical logic and algebra, "Nauka", Moscow, 1979. (Russian)

8. W. Sierpiński, Sur un problème concernant les fonctions semi-continues, Fund. Math. 28 (1937), $1-6$.

Mathematical Institute, University of Wroclaw, Pl. GRUNWAldzki 2/4, 50384 WROCLAW, POLAND 